Primljen / Received: 12.4.2017. Ispravljen / Corrected: 12.6.2017.

Prihvaćen / Accepted: 20.6.2017. Dostupno online / Available online: 10.2.2018.

\section{Method of incompatible modes - overview and application}

Authors:

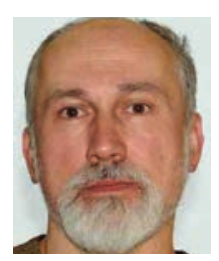

Prof. Ivica Kožar, PhD. CE

University of Rijeka

Faculty of Civil Engineering

ivica.kozar@gradri.uniri.hr

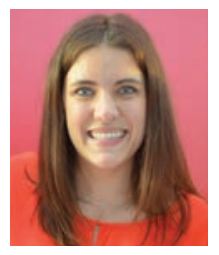

Tea Rukavina, MCE

University of Rijeka

Faculty of Civil Engineering

Université de Technologie de Compiègne

Sorbonne Universités, France

tea.rukavina@uniri.hr

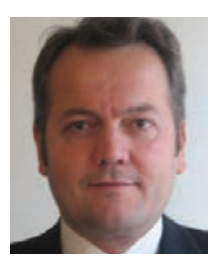

Prof. Adnan Ibrahimbegović, PhD. CE Université de Technologie de Compiègne Sorbonne Universités, France adnan.ibrahimbegovic@utc.fr

\section{Ivica Kožar, Tea Rukavina, Adnan Ibrahimbegović}

Subject review

\section{Method of incompatible modes - overview and application}

The finite-element method has been in use in the engineering community for over 50 years, a period during which it has been constantly improved. One important improvement is the addition of required displacement modes ("incompatible modes") into the element's shape functions. Such addition violates the continuity condition, and has to be realised according to certain rules if convergence is to be achieved. The benefits are shown in the element's behaviour under unfavourable loading conditions, and in the possibility of a simplified treatment of strain or displacement discontinuities.

Key words:
finite element, shape function, bending analysis, discontinuity analysis, static condensation

Pregledni rad

Ivica Kožar, Tea Rukavina, Adnan Ibrahimbegović

\section{Metoda nekompatibilnih modova - pregled i primjena}

Metoda konačnih elemenata koristi se u inženjerskoj zajednici već više od 50 godina i za to je vrijeme stalno poboljšavana. Jedno od bitnih poboljšanja odnosi se na uvođenje dodatnih funkcija za opisivanje pomaka ("nekompatibilnih modova") u interpolacije elemenata. Takvo dodavanje ne zadovoljava uvjet kontinuiteta i mora biti provedeno uz određene uvjete kako bi se postigla konvergencija. Prednosti korištenja ove metode jesu poboljšano ponašanje elemenata u nepovoljnim uvjetima opterećenja te mogućnost jednostavnog uzimanja u obzir diskontinuiteta u polju pomaka ili deformacija.

Ključne riječi:

konačni element, funkcija oblika, analiza savijanja, analiza diskontinuiteta, statička kondenzacija

Übersichtsarbeit

Ivica Kožar, Tea Rukavina, Adnan Ibrahimbegović

\section{Methode der inkompatiblen modi - übersicht und anwendung}

Die Finite-Elemente-Methode wird in der Ingenieurgemeinschaft seit mehr als 50 Jahre angewendet, und wurde während dieser Zeit ständig verbessert. Eines der wichtigsten Verbesserungen bezieht sich auf die Einführung zusätzlicher Funktionen für die Beschreibung der Verschiebung ("inkompatible Modi") bei der Interpolation der Elemente. Eine solche Ergänzung erfüllt nicht die Bedingung der Kontinuität und muss unter bestimmten Bedingungen durchgeführt werden, um eine Konvergenz zu erreichen. Die Vorteile der Anwendung dieser Methode liegen im verbesserten Verhalten der Elemente unter ungünstigen Belastungsbedingungen sowie der Möglichkeit einer einfachen Berücksichtigung der Diskontinuität im Bereich der Verschiebung oder Verformung.

Schlüsselwörter:

Finite-Elemente, Formfunktion, Biegeanalyse, Diskontinuitätsanalyse, statische Kondensation 


\section{Introduction}

It was observed already at initial stages of implementation that the greatest problem with finite elements with low number of nodes is their sensitivity to "locking" (e.g., see [1]). The remedy has been sought through enhancement of the displacement or strain element field, and the method was called "the method of incompatible modes" or simply "incompatible modes".

The method of incompatible modes was introduced in 1973 by Wilson et al. [2, 3] as an improvement of the low-order plane and solid elements in bending problems. It was observed through eigenvector analysis that low-order elements can not describe bending behaviour simply because they lack the necessary shape functions, and so the lacking displacement shapes were added artificially. Although simple addition of shape functions violates the continuity condition, the convergence can still be achieved if some requirements are met [4]. Incompatible shape functions are not unique, i.e. they assume various forms in different problems (small displacements, large displacements, large rotations, incompressible material, etc.). Many well performing incompatible shape functions belong to the class of mixed formulation elements [5].

It was established that this method has some additional benefits, such as an improved resistance to mesh distortions and improved behaviour of elements when nearly incompressible materials are analysed [6]. Also, incompatible modes are not only applied to improve the elements" behaviour, i.e. they can also be used to formulate strain or displacement based on constitutive laws (e.g., damage evolution) on the element level. In combination with the operator split technique (e.g., see [7]) that enables separate solution of evolution equations and equilibrium equations on the local and global levels, this drastically reduces demand for secondary storage. The application of incompatible modes to the geometrically nonlinear problem of large rotations can be found in $[8,9]$. Practical application of enhanced elements is possible in various areas, such as in the design of walls with openings [10].

The application of incompatible modes in strain and displacement discontinuities in 1D-problems is described in Section 2. The application of incompatible modes to the original problem of bending, where the behaviour of $2 \mathrm{D}$ and 3D quadrilateral elements in a structure loaded in bending has been improved, is described in Section 3.

\section{Incompatible mode method in 1D}

\subsection{Mixed variational formulation}

The standard finite element solution is usually constructed from the displacement type variational formulation, where the weak form is obtained only from equilibrium equation. If we want to enrich the strain field and introduce the enhanced approximation into the finite element formulation, we have to construct the weak form from all three sets of equations: kinematic, constitutive and equilibrium equations. This is called the mixed or Hu-Washizu variational formulation, where the displacement field $u(x)$, the strain field $\varepsilon(x)$, and the stress field $\sigma(x)$, are independent of one another [7]. ZTo be able to derive the method of incompatible modes, one should start by enriching the strain field in the following way:

$\varepsilon=\frac{d u}{d x}+\tilde{\varepsilon}$

where the first term is the standard strain, and the second term is the enhanced or incompatible strain.

In the weak form, equations no longer have to be satisfied at each point of the domain $\Omega$, but rather in an integral sense only. It is obtained by multiplying the equation by an arbitrary function $w$, also known as the weighting function, and then by integrating the product over the whole domain. For the equilibrium equation we get:

$\int_{\Omega} w\left(\frac{d \sigma}{d x}+b\right) d x=0$

which, after integrating by parts, assumes the following form:

$G_{u}=\int_{\Omega} \frac{d w}{d x} \sigma d x-\int_{\Omega} w b d x-[w \bar{t}]_{\Gamma_{\sigma}}=0$

Here, $w(x)$ represents the virtual displacement field that has to vanish on the boundary, thus satisfying the condition $w(x)=0$ on $\Gamma_{u^{\prime}}$ In a similar manner, the weak form of the constitutive equation is defined in the space of the virtual strain field $\gamma(x)$ for the case of linear elasticity:

$G_{\varepsilon}=\int_{\Omega} \gamma(E \varepsilon-\sigma) d x=0$

The weak form of the kinematic equation is constructed in the space of the virtual stress field $\tau(\mathbf{x})$ :

$G_{\sigma}=\int_{\Omega} \tau\left(\frac{d u}{d x}-\varepsilon\right) d x=0$

Eqs. (3), (4) and (5) constitute the mixed weak form that is equivalent to the Hu-Washizu functional [11] for a 1D case:

$\Pi(u, \varepsilon, \sigma)=\int_{\Omega} \frac{1}{2} \varepsilon E \varepsilon d x+\int_{\Omega} \sigma\left(\frac{d u}{d x}-\varepsilon\right) d x-\int_{\Omega} u b d x-[u \bar{t}]_{\Gamma_{\sigma}}$

The virtual strain field and the virtual stress field do not have to satisfy the boundary condition imposed on the virtual displacement field. Also, they do not have to be continuous, but square integrable functions only [7].

The enriched virtual strain field is defined in the same manner as the real strain field in Eq. (1):

$\gamma=\frac{d w}{d x}+\tilde{\gamma}$

By introducing the enriched approximations from Eqs. (1) and (7) into Eqs. (4) and (5), the following equations are obtained:

$G_{\varepsilon}=\int_{\Omega}\left(\frac{d w}{d x}+\tilde{\gamma}\right)\left[E\left(\frac{d u}{d x}+\tilde{\varepsilon}\right)-\sigma\right] d x=0$ 
$G_{\sigma}=\int_{\Omega} \tau \tilde{\varepsilon} d x=0$

Furthermore, Eq. (8) can be divided into two equations:

$\int_{\Omega} \frac{d w}{d x}\left[E\left(\frac{d u}{d x}+\tilde{\varepsilon}\right)-\sigma\right] d x=0$

$\int_{\Omega} \tilde{\gamma}\left[E\left(\frac{d u}{d x}+\tilde{\varepsilon}\right)-\sigma\right] d x=0$

From Eq. (10), it follows:

$\int_{\Omega} \frac{d w}{d x} \sigma d x=\int_{\Omega} \frac{d w}{d x} E\left(\frac{d u}{d x}+\tilde{\varepsilon}\right) d x$

and by introducing it into Eq. (3), we obtain:

$G_{u}=\int_{\Omega} \frac{d w}{d x} \sigma d x-\int_{\Omega} w b d x-[w \bar{t}]_{\Gamma_{\sigma}}=0$

Eqs. (9), (11) and (13) constitute the weak form for the enriched strain field obtained using the mixed formulation. They are listed here:

$\int_{\Omega} \frac{d w}{d x} \sigma d x-\int_{\Omega} w b d x-[w \bar{t}]_{\Gamma_{\sigma}}=0$

$\int_{\Omega} \tilde{\gamma}\left[E\left(\frac{d u}{d x}+\tilde{\varepsilon}\right)-\sigma\right] d x=0$

$\int_{\Omega} \tau \tilde{\varepsilon} d x=0$

\subsection{Finite element implementation}

A 2-node truss bar finite element of length $L$ and cross-sectional area $A$ is considered, with an axial degree of freedom $u_{i}$ at every node (see Figure 1).

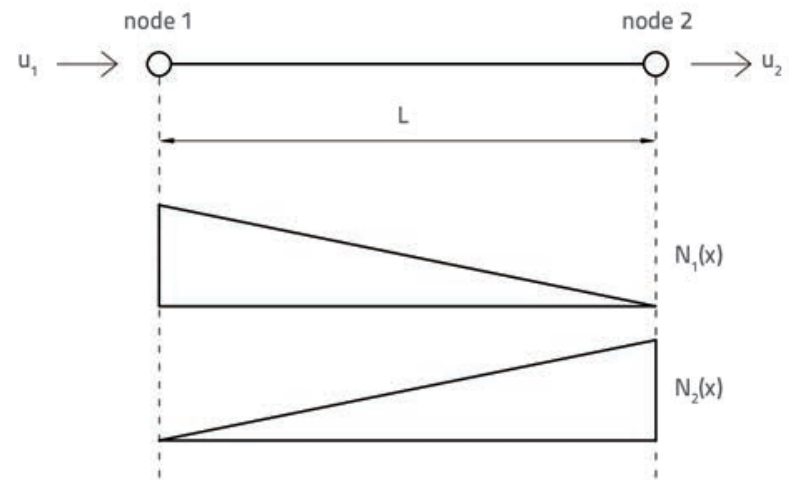

Figure 1. 2-node truss bar finite element with its shape functions

By introducing the finite element approximations, the enriched displacement field can be represented as the sum of the compatible and incompatible part:

$u(x)=\mathbf{N u}+M(x) \alpha$

where $\mathbf{N}$ are linear shape functions, $\mathbf{u}$ is the nodal displacement vector, $M(x)$ is the incompatible mode function, and $\alpha$ is the incompatible mode parameter. The incompatible mode function $M(x)$ is chosen according to the characteristics of the problem to be solved, as will be shown through examples in the following Sections.

The enriched strain field in Eq. (1) can be computed as the derivative of Eq. (15):

$\varepsilon(x)=\mathbf{B u}+G(x) \alpha$

where matrix B contains the shape functions" derivatives, and $\mathrm{G}(\mathrm{x})$ is the derivative of the incompatible mode function.

Besides the convergence conditions that have to be met by standard finite elements, several additional conditions have to be solved by enhanced elements with incompatible modes [7]: The incompatible strain field should be independent from the compatible strain field, and so their shape functions must not belong to the same space:

$N_{i} \cap M=\varnothing$

Stress parameters should be orthogonal to the incompatible mode parameters, to that the presence of stress in the approximation can be avoided. For this reason, all the terms in Eqs. (14) that contain stress parameters should vanish:

$\int_{\Omega} \tilde{\gamma} \sigma d x=0$
$\int_{\Omega} \tau \tilde{\varepsilon} d x=0$

Any incompatible strain should be orthogonal to any constant stress and no work should result from the coupling between these two fields. This ensures convergence in the spirit of the patch test. It has been shown in [3] that for a constant stress $\sigma$, it follows from Eqs. (18):

$\int_{\Omega} \bar{G}(x) d x=0 ; \quad \bar{G}=G-\frac{1}{\Omega} \int_{\Omega} \bar{G}(x) d x$

By introducing the finite element approximations for displacements and strains into the mixed formulation from Eqs. (14), a system of equations is obtained:

$$
\begin{aligned}
& \int_{\Omega} \mathbf{B}^{T} E A B \mathbf{u} d x+\int_{\Omega} \mathbf{B}^{T} E A G \alpha d x-\int_{\Omega} \mathbf{N} b A d x-[\mathbf{N} \overline{t A}]_{\Gamma_{\sigma}}=0 \\
& \int_{\Omega} G E A B^{T} \mathbf{u} d x+\int_{\Omega} G E A G \alpha d x=0
\end{aligned}
$$

When we introduce the substitutions:

$$
\begin{aligned}
& \mathbf{K}=\int_{\Omega} \mathbf{B}^{T} E A \mathbf{B} d x \\
& \mathbf{F}=\int_{\Omega} \mathbf{B}^{T} E A G d x \\
& \mathbf{H}=\int_{\Omega} G E A G d x \\
& \mathbf{f}=\int_{\Omega} \mathbf{N} b A d x-[\mathbf{N} \overline{t A}]_{\Gamma_{\sigma}}
\end{aligned}
$$


Eqs. (20) become:

$\mathbf{K u}+\mathbf{F}^{\top} \alpha=\mathbf{f}$

$\mathbf{F u}+\mathbf{H} \alpha=0$

and that can be written in matrix form:

$\left[\begin{array}{ll}\mathbf{K} & \mathbf{F}^{T} \\ \mathbf{F} & \mathbf{H}\end{array}\right]\left[\begin{array}{l}\mathbf{u} \\ \alpha\end{array}\right]=\left[\begin{array}{l}\mathbf{f} \\ 0\end{array}\right]$

Now the static condensation can be performed to reduce the stiffness matrix to the same size as the nodal displacement vector. From the second equation in Eqs. (22), it follows:

$$
\alpha=-\mathbf{H}^{-1} \mathbf{F u}
$$

When Eq. (24) is introduced into the first equation in Eqs. (22), the following is obtained:

$$
\left(\mathbf{K}-\mathbf{H}^{-1} \mathbf{F}^{T} \mathbf{F}\right) \mathbf{u}=\mathbf{f}
$$

where the first term represents the condensed stiffness matrix

$$
\hat{\mathbf{K}}=\mathbf{K}-\mathbf{H}^{-1} \mathbf{F}^{T} \mathbf{F}
$$

The following can finally be written:

$$
\hat{\mathbf{K}} \mathbf{u}=\mathbf{f}
$$

The method of incompatible modes derived in this section can be applied to a wide range of problems in mechanics. Two potential applications in a 1D setting will be shown in the following two subsections: the first one is a bar composed of two different materials, and the second one is a bar with an embedded discontinuity that simulates the crack opening.

\subsection{Examples}

\subsubsection{Strain discontinuity for a heterogeneous bar}

Consider a bar of length $L$ composed of two different materials, where the interface between them is located in the middle of the element $\bar{x}=\mathrm{L} / 2$.. Elastic moduli of the left and right part of the bar are marked as $E_{1}$ and $E_{2}$ such that $E_{1}>E_{2}$. The bar is clamped at the left end, with an imposed force $F$ on the right end, as can be seen in Figure 2. The following geometric and material properties are used: $E_{1}=1000, E_{2}=500, L=1, A=1$, and $F=300$.

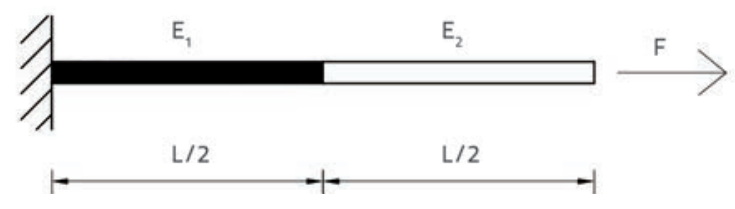

Figure 2. Heterogeneous bar composed of two different materials
Since the bar is composed of two different materials, there is a discontinuity in the strain field just in the middle of the element. The strain discontinuity is often called weak discontinuity, as opposed to the displacement discontinuity that is called strong discontinuity, and which will be explored in the next section. For an example where both strong and weak discontinuities have been implemented in one element see [12]. This example of a heterogeneous bar can easily be solved using two standard truss bar finite elements with different material properties. The values of nodal displacements can be obtained by simple calculation:

$u(x=L / 2)=0.15 ; u(x=L)=0.45$

Now, the same example can be solved using the method of incompatible modes described in the previous section. In this case, a single enhanced finite element is used.

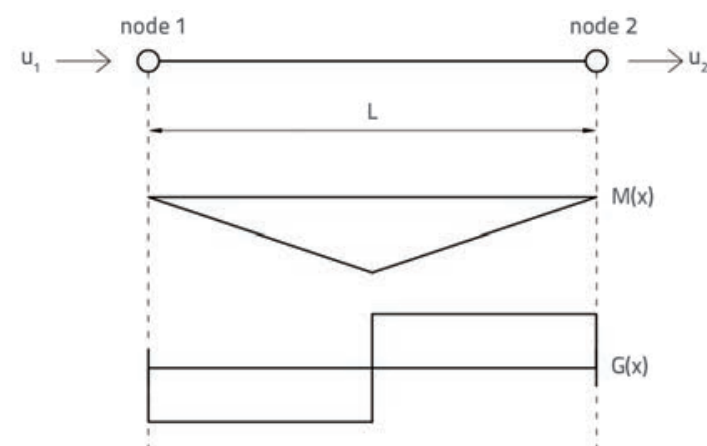

Figure 3. Incompatible mode function and its derivative for strain discontinuity modelling

The strain discontinuity is taken into account by choosing an appropriate incompatible mode function $\mathrm{M}(\mathrm{x})$, as shown in Figure 3 :

$M(x)=\left\{\begin{array}{cc}-\frac{2 x}{L}, & x \in[0, \bar{x}] \\ \frac{2(x-L)}{L}, & x \in[\bar{x}, L]\end{array}\right.$

The derivative of $M(x)$ is then:

$G(x)=\frac{d M(x)}{d x}= \begin{cases}-\frac{2}{L}, & x \in[0, \bar{x}] \\ \frac{2}{L}, & x \in[\bar{x}, L]\end{cases}$

Eqs. (21) have to be computed by dividing the domain $\Omega$ into two subdomains, $\Omega_{1} \in[0, \mathrm{~L} / 2]$ where $\mathrm{E}=\mathrm{E}_{1}$ and $\Omega_{2} \in[\mathrm{L} / 2, \mathrm{~L}]$ in which $\mathrm{E}=\mathrm{E}_{2}$ :

$$
\begin{aligned}
& \mathbf{K}=\int_{\Omega_{1}} \mathbf{B}^{T} E_{1} A \mathbf{B} d x+\int_{\Omega_{2}} \mathbf{B}^{T} E_{2} A \mathbf{B} d x \\
& \mathbf{F}=\int_{\Omega_{1}} \mathbf{B}^{T} E_{1} A G d x+\int_{\Omega_{2}} \mathbf{B}^{T} E_{2} A G d x \\
& \mathbf{H}=\int_{\Omega_{1}} G E_{1} A G d x+\int_{\Omega_{2}} G E_{2} A G d x \\
& \mathbf{f}^{T}=\left[\begin{array}{ll}
F & 0
\end{array}\right]
\end{aligned}
$$


After introduction of shape function values, Eqs. (31) become:

$\mathbf{K}=\left(E_{1}+E_{2}\right) \frac{A}{2 L}\left[\begin{array}{cc}1 & -1 \\ -1 & 1\end{array}\right]$

$\mathbf{F}=\left(E_{1}-E_{2}\right) \frac{A}{L}\left[\begin{array}{c}1 \\ -1\end{array}\right]$

$\mathbf{H}=\left(E_{1}+E_{2}\right) \frac{2 A}{L}$

from which the condensed stiffness matrix defined in Eq. (26) is obtained as follows:

$\mathbf{K}=\frac{E_{1} E_{2}}{E_{1}+E_{2}} \frac{2 A}{L}\left[\begin{array}{cc}1 & -1 \\ -1 & 1\end{array}\right]$

After taking into account boundary conditions, and after introduction of geometric and material parameters, Eq. (27) is solved to obtain displacement of the free end of the bar:

$u(x=L)=0.45$

To calculate the displacement at the discontinuity, it is first necessary to recover the value of the displacement jump $\alpha$. This is done via Eq. (24), from which we compute::

$\alpha=0.075$

The displacement at the discontinuity can be now calculated from Eq. (15):

$u(x=L / 2)=\mathrm{Nu}+M(x) \alpha=\frac{x}{L} u_{2}-\frac{2 x}{L} \alpha=0.15$

It has been shown here that, by using one finite element enhanced by an incompatible mode, it is possible to obtain the results similar to the ones already obtained from the calculation with two standard finite elements, given in Eq. (28).

\subsubsection{Displacement discontinuity for localized failure modelling}

The aim of this section is to present application of the method of incompatible modes to a softening damage model that is capable of representing failure of concrete in tension. When the limit strength is reached, a macro crack starts to develop, and so the softening occurs immediately after the linear elastic phase. There are several approaches for implementing softening into the model. The one described in $[7,12]$ is selected for the purposes of this paper. In the proposed model, an embedded strong discontinuity is introduced as a localized displacement jump that simulates the crack opening in the middle of the element. With this localization limiter, mesh-dependency is eliminated. There are other works in which a similar framework is used to describe the softening phase, either for damage, plasticity or coupled plasticity-damage models (e.g., in [14-17]). In damage, strain goes to zero after complete unloading, and unlike plasticity, there is no residual plastic strain.

The formulation for the simplest 1D truss bar finite element will be derived. The displacement discontinuity has to be introduced into kinematic equations, and it is implemented using the incompatible mode method.

To simulate the crack opening, a displacement jump $\alpha$ is introduced in the middle of the element $\bar{x}=\mathrm{L} / 2$. Since we are dealing with a truss bar finite element that has axial degrees of freedom only, this displacement jump can describe mode I crack opening only.

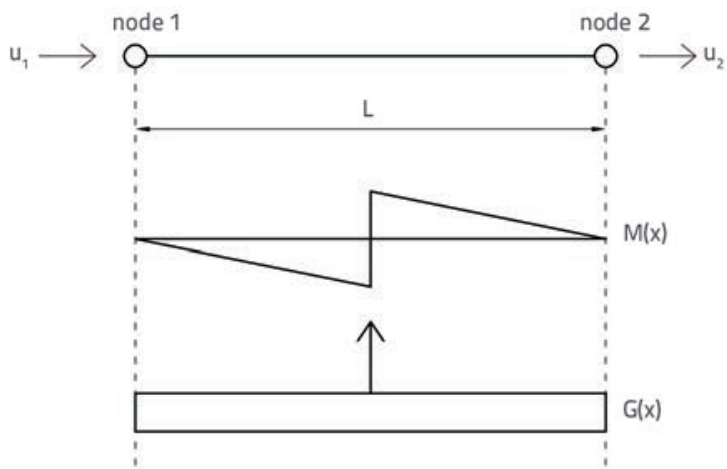

Figure 4. Incompatible mode function and its derivative for displacement discontinuity modelling

This localization can be introduced into the finite element formulation in the spirit of the incompatible mode method, as a displacement discontinuity. In this case, the incompatible mode function $M(x)$ shown in Figure 4 is chosen:

$M(x)=\left\{\begin{aligned}-\frac{x}{L}, & x \in[0, \bar{x}\rangle \\ 1-\frac{x}{L}, & x \in\langle\bar{x}, L]\end{aligned}\right.$

Here, the derivative of $\mathrm{M}(\mathrm{x})$ is defined as follows:

$G(x)=\tilde{G}+\delta_{\bar{x}}$

where $\tilde{G}=-1 / L$, and $\delta_{\bar{x}}$ is the Dirac delta function representing the singularpart of the strain field:

$\delta_{\bar{x}}=\left\{\begin{array}{l}\infty, x=x \\ 0, \text { otherwise }\end{array}\right.$

By introducing the enriched strain field approximation from Eq. (16) into Hooke"s law, the stress field of the bulk material is obtained, as defined in $[0, \bar{x} \cup \bar{x}, L]$, since $\delta_{\bar{x}}=0$ for $\mathrm{x} \neq \bar{x}$ :

$\sigma(x)=E(\mathbf{B u}+\tilde{G} \alpha)$

As behaviour at the discontinuity is inelastic, a 1D softening damage model is introduced. The energy dissipation is described 
by the traction-separation cohesive law (see Figure 5), defined by the following equation:

$t=\overline{\bar{D}}^{-1} \alpha$

where $\overline{\bar{D}}$ is the compliance modulus at the discontinuity that can take values from zero to infinity.

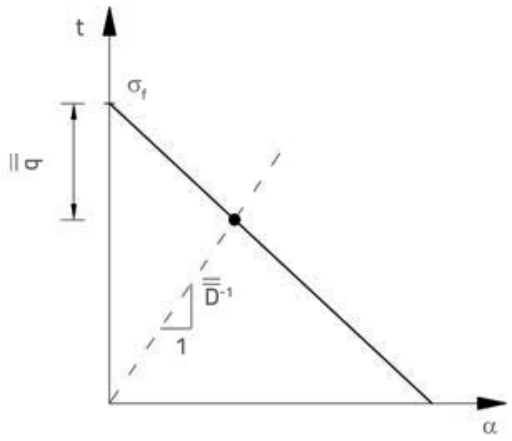

Figure 5. Cohesive law at the discontinuity

The damage function $\overline{\bar{\Phi}}$ checks if the value of traction at the discontinuity is admissible or not:

$\overline{\bar{\phi}}=|t|-\left(\sigma_{f}-\overline{\bar{q}}\right) \leq 0$

In Eq. (40), $\sigma_{f}$ is the elasticity limit, denoting the stress at which the first cracking occurs, and $\overline{\bar{q}}$ is the stress-like softening variable that controls damage defined by:

$\overline{\bar{q}}=-\overline{\bar{K}} \overline{\bar{\xi}}$

for linear softening. Here, $\overline{\bar{K}}$ is the softening modulus, and $\overline{\bar{\xi}}$ is the displacement-like softening variable that measures accumulation of damage.

The evolution of damage can be described by the softening damage multiplier $\bar{\gamma}$ that takes non-negative values only. The evolution equations for internal variables of this model are then:

$\dot{\overline{\bar{D}}}=\frac{\dot{\bar{\gamma}}}{t} ; \dot{\bar{\xi}}=\dot{\overline{\bar{\gamma}}} ; \dot{\alpha}=\dot{\overline{\bar{\gamma}}}$

and the loading/unloading conditions are listed here:

$\dot{\overline{\bar{\gamma}}} \geq 0 ; \overline{\bar{\phi}} \leq 0 ; \dot{\bar{\gamma}} \overline{\bar{\phi}}=0$

The damage consistency condition enforces the stress admissibility at subsequent time steps:

$\dot{\bar{\gamma}} \dot{\bar{\phi}}=0$

In practice, this means that there are two admissible cases:

Elastic case: $\overline{\bar{\phi}}<0, \dot{\bar{\gamma}}=0$

Damage case: $\overline{\bar{\phi}}=0, \dot{\bar{\gamma}}>0$
The fracture energy $G_{f}$ is equal to the area below the softening part of the response in Figure 6, with the slope equal to the softening modulus $\overline{\bar{K}}$ :

$\overline{\bar{K}}=-\frac{\sigma_{f}^{2}}{2 G_{f}}$

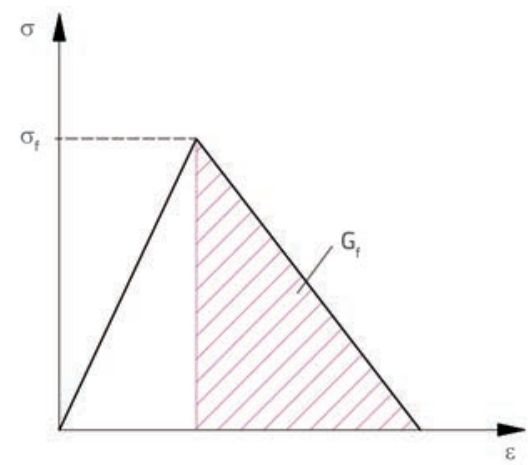

Figure 6. Fracture energy $G_{f}$

Numerical implementation of the presented model is quite complex, so that just a brief overview will be given in this paper. For a detailed and comprehensive description, please refer to [13]. Since evolution equations are defined locally, at the element level (at a Gauss quadrature point), and the equilibrium equations are defined globally, for the whole finite element mesh, the operator split methodology [7] will be used to attain computational efficiency. The computation is divided into two phases: local, where evolution equations of internal variables are considered, and global, where equilibrium equations are solved. The incremental and iterative Newton-Raphson procedure is used to find an approximate solution at each time step, while the implicit Backward Euler method is used to integrate the evolution equations.

In the local phase, the first step is the elastic trial step where the admissibility of the traction at the discontinuity is checked, as shown in Eq. (40). If $\overline{\bar{\Phi}} \leq 0$, the values are admissible and the step is indeed elastic, and if $\overline{\bar{\Phi}}>0$, the current step is not elastic and a correction must be made since the element has entered the softening phase. This is done by computing the positive value of the damage multiplier $\bar{\gamma}$, and by updating the softening variables $\overline{\bar{D}}$ and $\bar{\xi}$ and the displacement jump $\alpha$. Once the final values of internal variables are obtained, the global phase of the computation is initiated.

In the global phase, the check is made to determine if the equilibrium equations are satisfied within a chosen tolerance. If that is not the case, a new iterative sweep must be conducted to compute the updated values of nodal displacements. The system of equations to be solved is of the form as presented in Eq. (23), where the matrices $\mathbf{K}, \mathbf{F}$ and $\mathbf{H}$ are the same as in Eqs. (21), with an additional term in $\mathbf{H}$ related to the traction at the discontinuity:

$\mathbf{H}=\int_{0}^{L} \tilde{G} E A \tilde{G} d x+A \frac{\partial t}{\partial \alpha}$ 
A finite element computer code has been developed in Mathematica [18] and FEAP [19] to implement and validate the model. The chosen example is a cantilever bar clamped at the left-hand side, with the displacement $\bar{u}$ imposed at the righthand side (see Figure 7). The bar is discretized by two finite elements, where the material model for element 1 is softening damage, while it is linear elasticity for element 2 . The geometric and material properties used in this example are: $\mathrm{E}=1000, \sigma_{\mathrm{f}}$ $=150, \overline{\bar{K}}=-200, L=1$ and $A=1$. The imposed displacement is equal to $\bar{u}=0.75$.

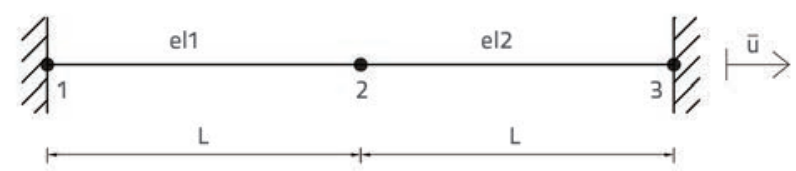

Figure 7. Cantilever bar with imposed displacement

The global response of the structure is shown in Figure 8. The behaviour of the bar is linear elastic until the elasticity limit is reached. Then the softening starts and the crack opens in the middle of element 1 . That means that all the stress is concentrated at the discontinuity, while the bulk of the material is unloading. If we unload the bar, the diagram goes back to zero, showing the typical behaviour of damage models. It can be seen that the unloading and the reloading follow the same path and, in the end, the bar reaches complete failure, $\sigma=0$.

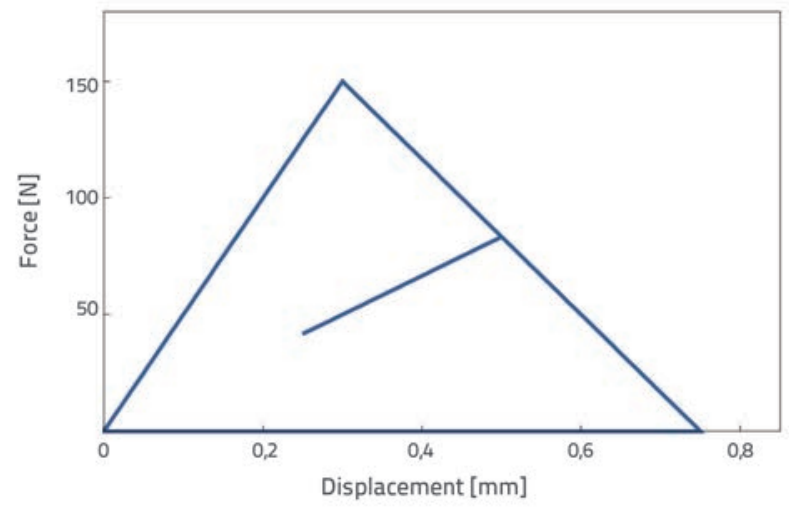

Figure 8. Force-displacement diagram with two loading-unloading cycles

\section{Incompatible mode method in 2D and 3D}

\subsection{D finite element}

\subsubsection{Formulation of Q4 element}

The formulation of the isoparametric quadrilateral 2D element is well known and one of many finite element references can be consulted in this respect, e.g., [4]. The geometry of the element is represented as follows $x(\xi, \eta)=N_{1}(\xi, \eta) x_{1}+N_{2}(\xi, \eta) x_{2}+\ldots+N_{r}(\xi, \eta) x_{r}=\sum_{i=1}^{r} N_{i}(\xi, \eta) x_{i}$

$y(\xi, \eta)=N_{1}(\xi, \eta) y_{1}+N_{2}(\xi, \eta) y_{2}+\ldots+N_{r}(\xi, \eta) y_{r}=\sum_{i=1}^{r} N_{i}(\xi, \eta) y_{i}$

where $\xi, \eta \in[-1,1]$ are isoparametric coordinates $N_{i}(\xi, \eta)$ are shape functions and $x_{i}, y_{i}$ are nodal coordinates, $i=(1,2,3,4)$, as presented in Figure 9.

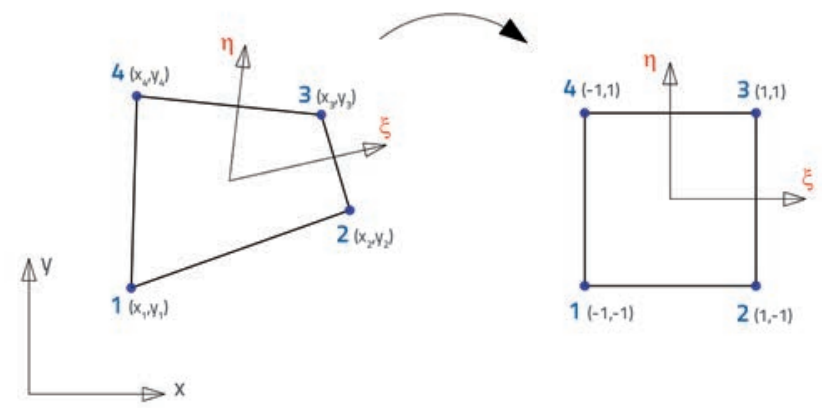

Figure 9. Isoparametric Q4 element

Displacements $\mathrm{u}$ and $\mathrm{v}$ are interpolated in the same manner

$u(\xi, \eta)=N_{1}(\xi, \eta) u_{1}+N_{2}(\xi, \eta) u_{2}+\ldots+N_{r}(\xi, \eta) u_{r}=\sum_{i=1}^{r} N_{i}(\xi, \eta) u_{i}$
$v(\xi, \eta)=N_{1}(\xi, \eta) v_{1}+N_{2}(\xi, \eta) v_{2}+\ldots+N_{r}(\xi, \eta) v_{r}=\sum_{i=1}^{r} N_{i}(\xi, \eta) v_{i}$

where $\mathrm{u}_{\mathrm{i}^{\prime}} \mathrm{v}_{\mathrm{i}}$ are nodal displacements, $\mathrm{i}=(1,2,3,4)$.

The geometry and displacement shape functions are equal for isoparametric finite elements. Isoparametric quadrilateral 2D element shape functions, in matrix notation, are

$\mathbf{N}(\xi, \eta)=\frac{1}{4}\left[\begin{array}{c}(1-\xi)(1-\eta) \\ (1+\xi)(1-\eta) \\ (1+\xi)(1+\eta) \\ (1-\xi)(1+\eta)\end{array}\right]$

The chosen shape functions result in element eigenvectors as depicted in Figure 10 (only two are shown as the other two are similar).
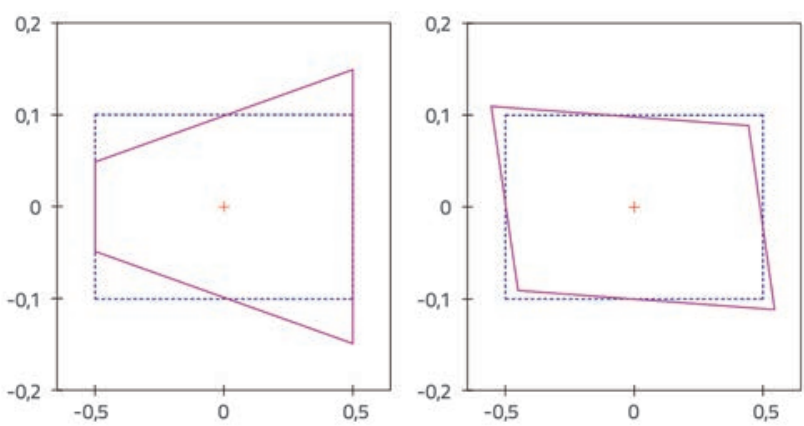

Figure 10. Characteristic Q4 element eigenvectors 
The relation between strains and displacements is as usual, and for a 2D plain stress / plane strain problem it reads as follows

$$
\boldsymbol{\varepsilon}=\left[\begin{array}{c}
\varepsilon_{x} \\
\varepsilon_{y} \\
\gamma_{x y}
\end{array}\right]=\left[\begin{array}{c}
\frac{\partial u}{\partial x} \\
\frac{\partial v}{\partial y} \\
\frac{\partial u}{\partial y}+\frac{\partial v}{\partial x}
\end{array}\right]=\mathbf{B u}
$$

with matrix $\mathbf{B}$ being defined as

$$
\mathbf{B}=\left[\begin{array}{cc}
\frac{\partial N_{i}}{\partial x} & 0 \\
0 & \frac{\partial N_{i}}{\partial y} \\
\frac{\partial N_{i}}{\partial y} & \frac{\partial N_{i}}{\partial x}
\end{array}\right] ; i=1, \ldots, 4
$$

The shape functions $N_{i}(\xi, \eta)$ depend on isoparametric coordinates $\xi, \eta$, and the derivatives are obtained by applying the chain rule for derivatives of composite functions

$\left[\begin{array}{l}\frac{\partial N}{\partial \xi} \\ \frac{\partial N}{\partial \eta}\end{array}\right]=\left[\begin{array}{ll}\frac{\partial x}{\partial \xi} & \frac{\partial y}{\partial \xi} \\ \frac{\partial x}{\partial \eta} & \frac{\partial y}{\partial \eta}\end{array}\right]\left[\begin{array}{l}\frac{\partial N}{\partial x} \\ \frac{\partial N}{\partial y}\end{array}\right]=\mathbf{J}\left[\begin{array}{l}\frac{\partial N}{\partial x} \\ \frac{\partial N}{\partial y}\end{array}\right]$

where $\mathbf{J}$ is the Jacobian matrix

$\mathbf{J}(\xi, \eta)=\left[\begin{array}{cc}\frac{\partial N_{i} x_{i}}{\partial \xi} & \frac{\partial N_{i} y_{i}}{\partial \xi} \\ \frac{\partial N_{i} x_{i}}{\partial \eta} & \frac{\partial N_{i} y_{i}}{\partial \eta}\end{array}\right]=\left[\begin{array}{c}\frac{\partial N_{i}}{\partial \xi} \\ \frac{\partial N_{i}}{\partial \eta}\end{array}\right]\left[\begin{array}{ll}x_{i} & y_{i}\end{array}\right] ; i=1, \ldots, 4$

The required partial derivatives of shape functions over global coordinates are finally

$\left[\begin{array}{c}\frac{\partial N_{i}}{\partial x} \\ \frac{\partial N_{i}}{\partial y}\end{array}\right]=\mathbf{J}^{-1}\left[\begin{array}{c}\frac{\partial N_{i}}{\partial \xi} \\ \frac{\partial N_{i}}{\partial \eta}\end{array}\right]$

In the case of small displacements and linear elasticity, the principle of minimum potential energy can be applied, and so the element stiffness matrix and the consistent load vector read

$$
\mathbf{K}=t \int_{A} \mathbf{B}^{T} \mathbf{D B} d A ; \mathbf{f}=t \int_{A} \mathbf{N}^{T} \mathbf{q} d A
$$

and the structure equilibrium equation is then $\mathbf{K} \mathbf{u}-\mathbf{f}=\mathbf{0}$. In Eq. (55), t is the element thickness, $\mathbf{D}$ is the elasticity tensor, and $\mathbf{q}$ is the surface load. Note: in the case where load is not applied on the element surface but on the element edge, the integral is over the line (the edge) and the shape matrix $\mathbf{N}$ is not complete.

We would like to transform our integrals into isoparametric coordinates, and so a change of variables is necessary $d A=|d \vec{\xi} \times d \vec{\eta}|=\operatorname{det}\left(\left[\begin{array}{ll}\frac{\partial x}{\partial \xi} & \frac{\partial y}{\partial \xi} \\ \frac{\partial x}{\partial \eta} & \frac{\partial y}{\partial \eta}\end{array}\right]\right) d \xi d \eta=|\mathbf{J}| d \xi d \eta$

and the stiffness matrix now reads

$\mathbf{K}=t \int_{-1}^{1} \int_{-1}^{1} \mathbf{B}^{T} \mathbf{D B}|\mathbf{J}| d \xi d \eta$

The actual integration is carried out numerically, i.e., applying the Gauss integration rule. In that case the integral is replaced with summation

$\mathbf{K}_{e l}=t \sum_{m=1}^{2} \sum_{n=1}^{2}\left(w_{m} w_{n} \operatorname{det}\left(\xi_{m}, \eta_{n}\right)_{e l} \mathbf{B}^{T}\left(\xi_{m}, \eta_{n}\right)_{e l} \mathbf{D B}\left(\xi_{m}, \eta_{n}\right)_{e l}\right)$

$\mathbf{P}_{e l}=t \sum_{m=1}^{2} \sum_{n=1}^{2}\left(w_{m} w_{n} \operatorname{det}\left(\xi_{m}, \eta_{n}\right)_{e l} \mathbf{N}^{\top}\left(\xi_{m}, \eta_{n}\right)_{e l} q\left(\xi_{m}, \eta_{n}\right)_{e l}\right)$

where $\mathrm{m}, \mathrm{n}$ represent the number of integration points along each isoparametric coordinate within the element, $w_{m^{\prime}} w_{n}$ are weighting coefficients and $\xi_{m^{\prime}} \eta_{n}$ are coordinates of the Gauss integration points.

\subsubsection{Addition of incompatible modes}

Figure 10 shows that the described Q4 element cannot describe bending very well (when the opposite sides rotate as in bending, the other two remain straight and not curved and so the spurious shear stress develops making the element unrealistically stiff). This might be compensated with the addition of the missing displacements of quadratic type

$\left[\begin{array}{l}u \\ v\end{array}\right]=\left[\begin{array}{l}N_{i} I \\ N_{i} I\end{array}\right]\left[\begin{array}{l}u_{i} \\ v_{i}\end{array}\right]+\left[\begin{array}{l}M_{j} I \\ M_{j} I\end{array}\right]\left[\begin{array}{l}\alpha_{j} \\ \alpha_{j}\end{array}\right] ; i=1, \ldots, 4 ; j=1, \ldots, 2$

where $\alpha_{1}, \alpha_{2}$ are the incompatible mode parameters to be determined, and the incompatible modes shape functions are $M_{1}=1-\xi^{2}$ and $M_{2}=1-\eta^{2}$. Displacements in the direction $\xi$ corresponding to the newly added functions (incompatible modes) are shown in Figure 11 (red crosses represent internal element points so the change in "density" is visible).
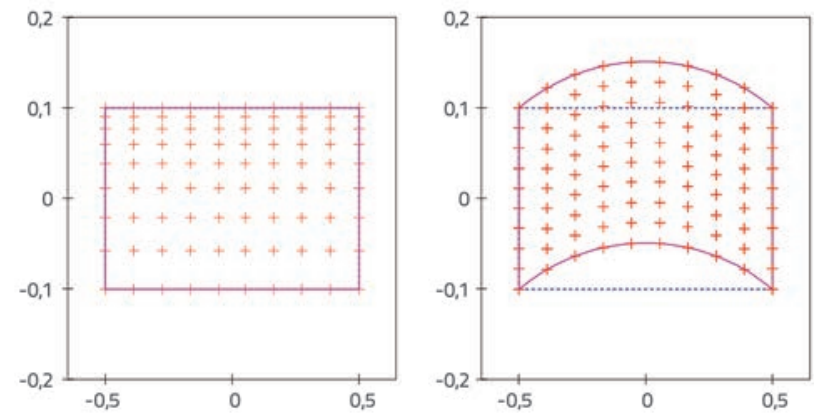

Figure 11. Characteristic Q4 element eigenvectors after addition of incompatible modes 
Thestrain-displacementmatrix corresponding to the incompatible modes is formulated so that the patch test condition from Eq.

(19) is satisfied, where $\mathbf{G}$ for the Q4 element is

$\mathbf{G}_{j}=\left[\begin{array}{c}\frac{\partial M_{j}}{\partial x} \\ \frac{\partial M_{j}}{\partial y} \\ \frac{\partial M_{j}}{\partial x}+\frac{\partial M_{j}}{\partial y}\end{array}\right] ; \quad j=1, \ldots, 2$

The complete $\mathbf{B}$ matrix with the added incompatible modes is now $\mathbf{B}_{\mathrm{m}}=[\mathbf{B} \mathbf{G}]$, and the stiffness matrix formulation proceeds as usual, with $\mathbf{B}$ matrix replaced by $\mathbf{B}_{\mathrm{m}}$ matrix

$\mathbf{K}_{m}=t \int_{A} \mathbf{B}_{m}^{T} \mathbf{D B}{ }_{m} d A$

In this case, the displacement vector $\mathbf{u}$ and the external force vector also have to be augmented with the incompatible mode parameters $\alpha$, and so we obtain

$\mathbf{u}_{m}=\left[\begin{array}{l}\mathbf{u} \\ \boldsymbol{\alpha}\end{array}\right] ; \mathbf{f}_{m}=\left[\begin{array}{l}\mathbf{f} \\ \mathbf{0}\end{array}\right]$

We now have a system of equations like Eq. (23), that reads

$\mathbf{K}_{m} \mathbf{u}_{m}=\mathbf{f}_{m}$

The thus formed new element is named Q6.

\subsubsection{Example}

The behaviour of the new Q6 element can be observed in the example of a cantilever beam loaded in bending. Figure 12 shows that there is a force and a moment loading. This is not a real patch test, however. It can be seen in [9] that both $2 \mathrm{D}$ and $3 \mathrm{D}$ incompatible quad elements pass the patch test. The figure is not proportional, it is a thin cantilever, so that the exact solution is easily calculated; the data is $\mathrm{E}=3 \mathrm{E} 7, \mathrm{v}=0.33$ (Poisson's ratio), plane stress, $L=3.0, h=0.2$ (length/height ratio is 15 ). The comparison of results without and with incompatible modes is shown in the following table:

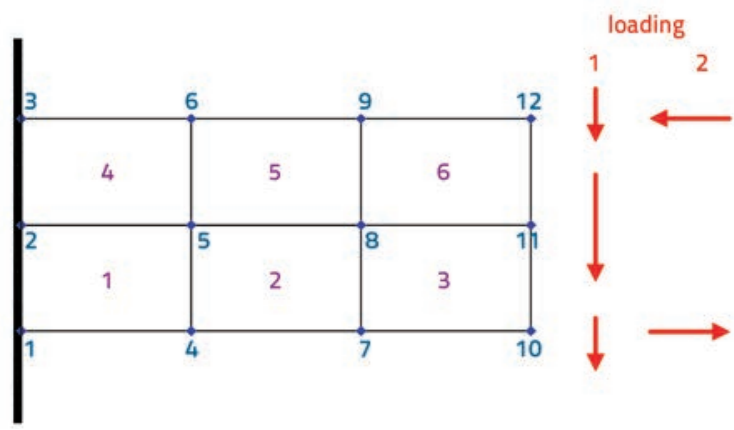

Figure 12. Cantilever beam and its loading
Table 1. Comparison of displacement results in 2D

\begin{tabular}{|c|c|c|c|}
\hline Displacements & exact & without IM & with IM \\
\hline Loading 1 & 0.562 & 0.169 & 0.544 \\
\hline Loading 2 & 0.112 & 0.033 & 0.113 \\
\hline
\end{tabular}

Graphical comparison of "standard" and "incompatible modes" of Q4 elements is presented in Figure 13.

Stresses in elements with incompatible nodes are recovered in the same way as for elements without incompatible modes.
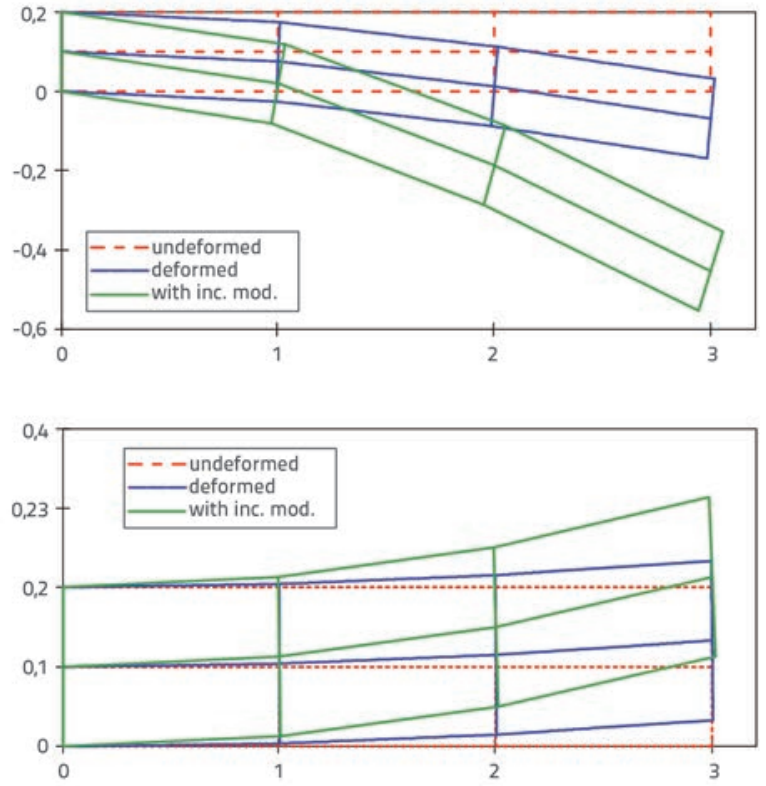

Figure 13. Comparison of deflections under force and moment loading

\subsection{D finite element}

\subsubsection{Formulation of $Q 8$ element}

In principle, the Q8 element is equal to the Q4 element but expanded in the third dimension, so there are more degrees of freedom. Due to this fact, the formulation of the Q8 element will only be briefly exposed. The isoparametric approximation for the geometry and displacements inside an element is

$\mathbf{x}=\mathbf{N} \mathbf{x}_{i} ; \mathbf{u}=\mathbf{N u}_{i} ; i=1, \ldots, 8$

where shape interpolation functions are standard isoparametric functions (see [4]), and so the interpolation matrix element $\mathbf{N}$ is

$N_{i}=\frac{1}{8}\left(1+\xi \xi_{i}\right)\left(1+\eta \eta_{i}\right)\left(1+\zeta \zeta_{i}\right) ; \mathbf{N}_{i}=N_{i} \mathbf{I}, i=1, \ldots, 8$

where $\xi_{\mathrm{j}^{\prime}} \eta_{\mathrm{i}^{\prime}} \zeta_{\mathrm{i}}$ are nodal isoparametric coordinates.

Equations for the strain-displacement matrix $\mathbf{B}$, stiffness matrix $\mathbf{K}$, and consistent loading matrix $\mathbf{f}$ are similar to those for the Q4 element. 


\subsubsection{Addition of incompatible modes}

The Q8 element suffers from the same lack of bending shapes as the Q4, and the remedy is based on the same idea: the addition of the missing shapes.

$\left[\begin{array}{c}u \\ v \\ w\end{array}\right]=\left[\begin{array}{c}N_{i} \mathbf{I} \\ N_{i} \mathbf{I} \\ N_{i} \mathbf{I}\end{array}\right]\left[\begin{array}{c}u_{i} \\ v_{i} \\ w_{i}\end{array}\right]+\left[\begin{array}{c}M_{j} \mathbf{I} \\ M_{j} \mathbf{I} \\ M_{j} \mathbf{I}\end{array}\right]\left[\begin{array}{c}\alpha_{j} \\ \alpha_{j} \\ \alpha_{j}\end{array}\right] ; i=1, \ldots, 8 ; j=1,2,3$

where the incompatible mode shape functions are defined as follows

$M_{1}=\left(1-\xi^{2}\right) ; M_{2}=\left(1-\eta^{2}\right) ; M_{3}=\left(1-\zeta^{2}\right) ;$

The strain-displacement matrix is defined as $\mathbf{B}_{\mathrm{m}}=[\mathbf{B} \mathbf{G}]$, where matrices $\mathbf{B}$ and $\mathbf{G}$ for the Q8 element are

$\mathbf{B}_{i}=\left[\begin{array}{c}\frac{\partial N_{i}}{\partial x} \mathbf{I} \\ \frac{\partial N_{i}}{\partial y} \mathbf{I} \\ \frac{\partial N_{i}}{\partial z} \mathbf{I}\end{array}\right] ; \mathbf{G}_{j}=\left[\begin{array}{c}\frac{\partial M_{j}}{\partial x} \mathbf{I} \\ \frac{\partial M_{j}}{\partial y} \mathbf{I} \\ \frac{\partial M_{j}}{\partial z} \mathbf{I}\end{array}\right] ; i=1, \ldots, 8 ; j=1,2,3$

The system of equations is again formed as in Eq. (64).

\subsubsection{Example}

The analysis is performed for the structure in Figure 14 with the data $\mathrm{E}=3 \mathrm{E} 7, v=0.33$, plane stress, $\mathrm{L}=3.0, \mathrm{~h}=0.2$, and $\mathrm{t}=$ 0.1 . The structure is the same as in the $2 \mathrm{D}$ example for the $\mathrm{Q} 4$ element, and so the comparison between elements is possible. The results of the analvsis are presented in Table 2.

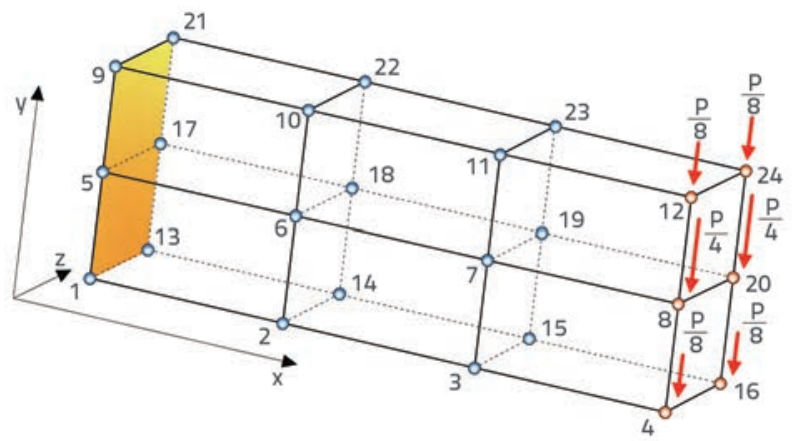

Figure 14. Example of 3D cantilever beam with loading
Table 2. Comparison of displacement results in 3D

\begin{tabular}{|c|c|c|c|}
\hline Displacements & exact & without IM & with IM \\
\hline Loading 1 & 0.562 & 0.167 & 0.544 \\
\hline Loading 2 & 0.112 & 0.033 & 0.112 \\
\hline
\end{tabular}

The comparison with the Q4 element shows that displacements due to the force are slightly less accurate, while the accuracy of the displacements due to the moment is about the same. It should be noted that the Q8 element is numerically much more complex: the element stiffness matrix is $24 \times 24$ and with incompatible modes (without condensation) it is $32 \times 32$, compared to $8 \times 8$ and $12 \times 12$ for the Q4 element.

\section{Conclusion}

This work presents a brief overview of the method of incompatible modes. The authors have placed them into two common areas of application: failure analysis and enhancement of behaviour. In the context of failure analysis, incompatible modes have been used to introduce displacement and strain functions whose parameters enable description of failure within the element itself. The procedure has been demonstrated on a $1 \mathrm{D}$ element for both strain and displacement discontinuities.

In the context of enhancement of the finite elements" capabilities, incompatible modes have been used to enrich the field of available displacements of standard shape functions. Additional quadratic shapes that have been added greatly improve the bending behaviour of such elements. Both 2D and 3D examples are presented.

It has been demonstrated that the method of incompatible modes has good properties in both areas of application and that its use should be encouraged. Additional degrees of freedom could be eliminated through condensation, or reduced through the use of the operator split procedure. Results obtained using incompatible modes are superior to the ones involving "standard" finite elements, i.e., comparable results could only be obtained using a much higher number of "un-enhanced" finite elements.

\section{Acknowledgements}

This work was supported by the Croatian Science Foundation Grant No. 9068 Multi-scale concrete model with parameter identification, and by the French Embassy in Croatia through the French Government scholarship for doctoral level. The support is gratefully acknowledged. 


\section{REFERENCES}

[1] Wilson, E.L., Ibrahimbegovic, A.: Addition of incompatible displacement modes for the calculation of element stiffness and stress, Finite Elements In Analysis and Design, 7 (1990), pp. 229242, https://doi.org/10.1016/0168-874X(90)90034-C

[2] Wilson, E.L., Taylor, R.L., Doherty, W.P., Ghaboussi, J.: Incompatible displacement models, In Fenves, S.J., Perrone, N., Robinson, A.R., Schnobrich, C. (editors), Numerical and Computer Methods in Structural Mechanics, Academic Press, pp. 43-57, 1973, https://doi. org/10.1016/B978-0-12-253250-4.50008-7

[3] Ibrahimbegovic, A., Wilson, E.L.: A modified method of incompatible modes, Communications in Applied Numerical Methods, 7 (1991), pp. 187-194, https://doi.org/10.1002/cnm.1630070303

[4] Zienkiewicz, O.C., Taylor, R.L., Zhu, J.Z.: The finite element method: its basis and fundamentals, 7th edition, Butterworth-Heinemann, 2013.

[5] Simo, J.C., Rifai, M.S.: A class of mixed assumed strain methods and the method of incompatible modes, International Journal for Numerical Methods in Engineering, 29 (1990), pp. 1595-1638, https://doi.org/10.1002/nme.1620290802

[6] Gharzeddine, F., Ibrahimbegovic, A.: Incompatible mode method for finite deformation quasi-incompressible elasticity, Computational Mechanics, 24 (2000), pp.419-425, https://doi. org/10.1007/s004660050001

[7] Ibrahimbegovic, A.: Nonlinear solid mechanics, theoretical formulations and finite element solution methods, Springer, 2009.

[8] Ibrahimbegovic, A., Frey, F.: Geometrically nonlinear method of incompatible modes in application to finite elasticity with independent rotation field, International Journal for Numerical Methods in Engineering, 36 (1993), pp. 4185-4200, https://doi. org/10.1002/nme.1620362406

[9] Kozar, I., Ibrahimbegovic, A.: Finite element formulation of the finite rotation solid element, Finite Elements in Analysis and Design, 20 (1995) 2, pp. 101-126, https://doi.org/10.1016/0168$874 \times(95) 00014-K$

[10] Fresl, K., Lazarević, D., Hak, S., Šavor Novak, M.: On the design of walls with openings, GRAEEVINAR, 67 (2015) 2, pp. 103-121.
[11] Washizu, K.: Variational methods in elasticity and plasticity, 2nd edition, Pergamon Press, 1975.

[12] Ibrahimbegovic, A., Melnyk, S.: Embedded discontinuity finite element method for modeling of localized failure in heterogeneous materials with structured mesh: an alternative to extended finite element method, Computational Mechanics, 40 (2007) 1, pp.149155, https://doi.org/10.1007/s00466-006-0091-4

[13] Jukic, M., Brank, B., Ibrahimbegovic, A.: Failure analysis of reinforced concrete frames by beam finite element that combines damage, plasticity and embedded discontinuity, Engineering Structures, 75 (2014), pp. 507-527, https://doi.org/10.1016/j. engstruct.2014.06.017

[14] Brancherie, D.: Modeles continus et discrets pour les problemes de localisation et de rupture fragile et ou ductile, PhD thesis, Ecole Normale Superieure de Cachan, 2003.

[15] Dominguez, N., Brancherie, D., Davenne, L., Ibrahimbegovic,A.: Prediction of crack pattern distribution in reinforced concrete by coupling a strong discontinuity model of concrete cracking and a bond-slip of reinforcement model, Engineering Computations, 22 (2005) 5-6, pp. 558-582, https://doi. org/10.1108/02644400510603014

[16] Ibrahimbegovic, A., Boulkertous, A., Davenne, L., Brancherie, D.: Modelling of reinforced-concrete structures providing crackspacing based on X-FEM, ED-FEM and novel operator split solution procedure, International Journal for Numerical Methods in Engineering, 83 (2010) 4, pp. 452-481, https://doi.org/10.1002/ nme.2838

[17] Ibrahimbegovic, A., Brancherie, D.: Combined hardening and softening constitutive model of plasticity: precursor to shear slip line failure, Computational Mechanics, 31 (2003) 1-2, pp. 88-100, https://doi.org/10.1007/s00466-002-0396-x

[18] Inc. Wolfram Research: Mathematica, 2015.

[19] Taylor, R.L.: FEAP - Finite Element Analysis Program, 2014. 